A\&A 365, 37-48 (2001)

DOI: $10.1051 / 0004-6361: 20000012$

(c) ESO 2001

\title{
Dust in active nuclei
}

\section{Powder or gravel?}

\author{
R. Maiolino, A. Marconi, and E. Oliva
}

Osservatorio Astrofisico di Arcetri L.go E. Fermi 5, 50125 Firenze, Italy

Received 17 July 2000 / Accepted 28 September 2000

\begin{abstract}
In a companion paper, Maiolino et al. (2001) presented various observational evidences for "anomalous" dust properties in the circumnuclear region of AGNs and, in particular, the reduced $E_{B-V} / N_{\mathrm{H}}$ and $A_{V} / N_{\mathrm{H}}$ ratios, the absence of the silicate absorption feature in mid-IR spectra of Sy2s and the absence of the carbon dip in UV spectra of reddened Sy1s. In this paper we discuss various explanations for these facts. The observational constraints favor a scenario where coagulation, catalyzed by the high densities in the circumnuclear region, yields to the formation of large grains. The resulting extinction curve is featureless, flatter than Galactic and the $E_{B-V} / N_{\mathrm{H}}$ and $A_{V} / N_{\mathrm{H}}$ ratios are significantly reduced. These results should warn about an inappropriate use of the standard Galactic extinction curve and $A_{V} / N_{\mathrm{H}}$ ratio when dealing with the extreme gas conditions typical of the circumnuclear clouds of AGNs. We also investigated alternative scenarios for the observed anomalous properties of dust in AGNs. Some of these scenarios might explain some of the observed properties for a few objects, but they generally fail to account for all of the observational constraints obtained for the large sample of AGNs studied in these works.
\end{abstract}

Key words. galaxies: Seyfert - galaxies: nuclei - galaxies: ISM - dust, extinction

\section{Introduction}

Some authors in the past have found evidences for anomalous properties of the dust in the circumnuclear region of AGNs. In particular, dust absorption and reddening appear to be systematically lower than expected from the gaseous column density, for a Galactic gas-to-dust ratio and extinction curve (e.g. Maccacaro et al. 1982; Reichert et al. 1985; Granato et al. 1997). In a companion paper (Maiolino et al. 2001, Paper I) we have strengthened these previous results and provided additional evidences that the dust in the circumnuclear region of AGNs is characterized by anomalous properties. By comparing the reddening toward the Broad Line Region (BLR) and the gaseous $N_{\mathrm{H}}$ inferred from the X-rays in a sample of 19 AGNs we found that, with the exception of three Low Luminosity AGNs, the $E_{B-V} / N_{\mathrm{H}}$ ratio is lower than the Galactic standard value by a factor ranging from $\sim 3$ to $\sim 100$. By comparing the optical and X-ray properties of various AGN classes (type 1.8-1.9 Sys, hard X-ray selected and radio selected QSOs, Broad Absorption Line QSOs and grism-selected QSOs) it was inferred that the $A_{V} / N_{\mathrm{H}}$ ratio must be one or two orders of magnitude lower than Galactic. Despite the large mid-IR continuum absorption inferred from the PAHs equivalent width, the absence

Send offprint requests to: R. Maiolino,

e-mail: maiolino@arcetri.astro.it of a significant silicate absorption feature at $9.7 \mu \mathrm{m}$ in the average ISO spectrum of Sy2s indicates that dust grains in the circumnuclear region of AGNs have different properties with respect to the Galactic diffuse interstellar medium. Finally, the lack of the $2175 \AA$ carbon dip in the UV spectra of some type 1 AGNs, which appear affected by dust reddening, suggests a depletion of small grains in the absorbing medium, as mentioned in Paper I. These findings suggest that the dust grain distribution is biased in favor of large grains. The presence of large grains in the circumnuclear region of AGNs was also suggested by Laor \& Draine (1993) to explain some of the infrared properties of AGNs.

In this paper we examine some possible explanations of the observational findings presented in Paper I. In particular, we compare our models with the quantitative constraints given on the $E_{B-V} / N_{\mathrm{H}}$ ratio, the equivalent width of the silicate feature and the depth of the $2175 \AA$ carbon dip. Note that we often distinguish the $E_{B-V} / N_{\mathrm{H}}$ and $A_{V} / N_{\mathrm{H}}$ ratios since, when discussing extinction curves different from Galactic, reddening and absorption are no longer tied by the standard Galactic relation $A_{V} / E_{B-V}=3.1$. In other words, models which could account for a decreasing of $E_{B-V} / N_{\mathrm{H}}$ do not necessarily lower the $A_{V} / N_{\mathrm{H}}$ ratio, and vice versa.

In Sect. 2 we first analyze and, in practice, dismiss scenarios with a standard (Galactic) grain size distribution. 
The case for large grains is analyzed and discussed in Sect. 3 while in Sect. 4 we draw our conclusions.

\section{The case for standard grains}

\subsection{Metallicity effects}

Various studies have found evidence for super-solar metallicities in the nuclear region of AGNs (Ferland et al. 1996; Hamann \& Ferland 1993, 1999; Fosbury et al. 1999; Reynolds et al. 1995; Dietrich \& Wilhelm-Erkens 2000).

The gaseous column densities used in Paper I were inferred from the photoelectric cutoff observed in the X-rays, but actually such a cutoff gives a measure of the column of metals $N_{\mathrm{Z}}$ (Morrison \& McCammon 1983), which is then converted into hydrogen column $N_{\mathrm{H}}$ by assuming solar abundances. If the metallicity is higher than solar, then the $N_{\mathrm{H}}$ inferred from the X-rays would be overestimated. On the other hand, a large fraction of refractory elements is locked into grains. As a consequence, an increased metallicity would also imply a higher dust content. Both the dust content and the $N_{\mathrm{Z}}$ to $N_{\mathrm{H}}$ conversion factor depend linearly on the metallicity and, therefore, any change in metallicity should not affect the $E_{B-V} / N_{\mathrm{H}}$ ratio in the way we measure it. Finally, we should emphasize that there are no obvious astrophysical nucleosynthesis processes that favor the formation of non-refractory elements over refractory ones, implying that even "peculiar" metallicities (in terms of relative abundances between metals) should not affect the observed $E_{B-V} / N_{\mathrm{H}}$ ratio.

Also, metallicity effects cannot explain the discrepancy between the lack of silicate feature and the strong mid-IR absorption in Sy2s. In the Small Magellanic Cloud the lack of the $2175 \AA$ carbon dip is ascribed to a low metallicity, but this is unlikely to be the explanation for AGNs since, as discussed above, generally the latter are characterized by super-solar metallicities.

Although metallicity effects cannot explain the anomalous properties of dust in AGNs, it can have important consequences on the expected emission line fluxes in the case of ionized gas containing dust. This is an issue which will be discussed further in Sects. 2.6 and 3 .

\subsection{Reflected broad lines}

Another possibility is that the broad lines used to measure $E_{B-V}$ in Paper I are not observed through the same medium that absorbs the X-rays (the obscuring torus), but are scattered by a medium (mirror) observed through a column density much lower than along the line of sight of the X-ray source. In this case the nuclei of these objects are expected to show significant polarization. Table 1 gives the optical and/or near-IR polarization for the objects examined in Paper I (therein Table 1) for which this information is available. For most of them the polarization is $<2 \%$, at least in the wavebands where the reddening is estimated (or as directly indicated by the polarization of $\mathrm{H} \alpha$ in two cases). For NGC 5506, NGC 2992 and Mkn 231,
Table 1. Percentage of polarization in the optical $(B, V)$ and in the near-IR $(\mathrm{H})$ for the subsample of the sources listed in Table 1 of Paper I for which this information is available. Polarization fraction in parenthesis are corrected for dilution of the stellar continuum. Notes: ${ }^{a}$ transm.- objects for which there is substantial evidence that most of the polarization is due to dichroic transmission; $P_{\mathrm{H} \alpha}-$ gives the polarization percentage of the broad $\mathrm{H} \alpha$ line. References: 1) Barth et al. (1999), 2) Young et al. (1996), 3) Kay (1994), 4) Brindle et al. (1990), 5) Berriman et al. (1989), 6) Goodrich (1992), 7) Corbett et al. (1998), 8) Wills et al. (1992), 9) Goodrich \& Miller (1994)

\begin{tabular}{lccccc}
\hline Name & $P_{\mathrm{B}}$ & $P_{\mathrm{V}}$ & $P_{\mathrm{H}}$ & Comm. $^{a}$ & Refs. \\
\hline M 81 & & 0.36 & & & 1 \\
NGC 1365 & 1.19 & 0.91 & 0.11 & & 4 \\
MCG-5-23-16 & 0.42 & $<0.8$ & 0.7 & & 4 \\
NGC 5506 & 3.10 & 2.35 & 2.44 & transm. & 2,5 \\
NGC 2992 & $2.62(15.9)$ & 3.1 & 1.18 & transm. & $3,4,6$ \\
Mkn6 & 0.5 & & & & 5 \\
3C 445 & & 2.1 & 0.75 & $P_{\mathrm{H} \alpha}=1.0$ & 4,7 \\
PG 2251+113 & 1.0 & & & & 8 \\
IRAS 1319-16 & $1.1(7.4)$ & & & $P_{\mathrm{H} \alpha}=0.2$ & 2,3 \\
NGC 526a & 0.26 & & & & 5 \\
Mkn 231 & 7.3 & 4.2 & 0.6 & transm. & 2,9 \\
\hline
\end{tabular}

which show higher polarization, there are evidences that most of the polarization is not due to reflection but to dichroic transmission.

Also, the scattering efficiency generally does not exceed a few percent. As a consequence, we would expect that the broad lines observed in our objects, once corrected for the observed reddening, are underluminous by a factor of $\sim 100$ with respect to the intrinsic luminosity indicated by the absorption-corrected X-ray luminosity, when compared to unobscured type 1 Seyferts and QSOs. Figure 1 shows the broad $\mathrm{H} \alpha$ luminosity, reddening-corrected assuming a Galactic standard extinction curve, versus the intrinsic X-ray luminosity, again for the objects listed in Table 1 of Paper I. The solid line and the shaded region show the $L_{\mathrm{H} \alpha}$ vs. $L_{\mathrm{X}}$ relation (mean and $\pm 1 \sigma$ dispersion respectively) derived for a large sample of PG QSOs and nearby Sy1s not showing evidence for absorption. Clearly none of the objects is underluminous in broad $\mathrm{H} \alpha$ by the factor of $\sim 100$ expected in the case of reflection. The most "H $\alpha$-deficient" object is NGC 2992. This object does show significant polarization (Table 1), but as discussed above this is very likely a dichroic effect due to transmission through the host galaxy, which is seen edge-on; an interpretation which is also supported by the finding that the [OIII] line is polarized at the same level as the continuum and broad $\mathrm{H} \alpha$ line (Goodrich 1992). As we shall discuss in Sect. 3, the most likely interpretation for the $\mathrm{H} \alpha$ deficiency in this object is a peculiar extinction curve. It should also be noted that Mkn 231 is actually significantly overluminous in $\mathrm{H} \alpha$, by a factor of $\sim 30$. This object is actually known to be underluminous in the X-rays. Possibly, the absorbing column density might be 


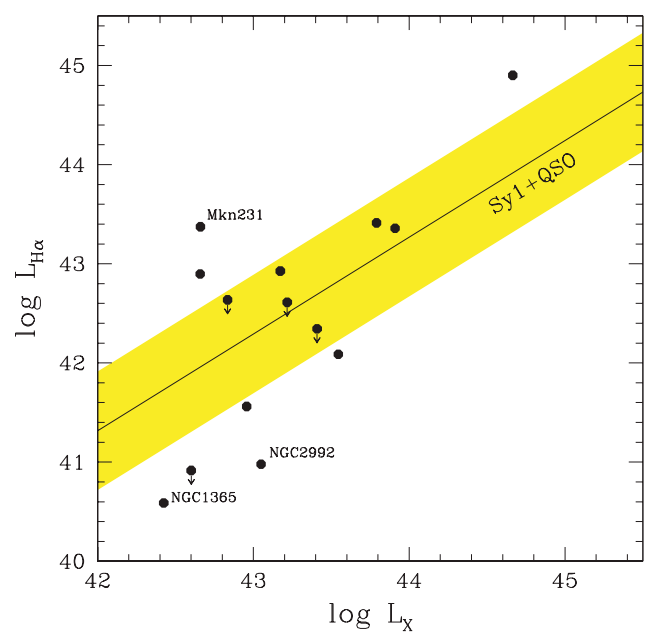

Fig. 1. H $\alpha$ luminosity for the objects reported in Table 1 of Paper I, corrected for the reddening derived by assuming a Galactic extinction curve, as a function of the $2-10 \mathrm{keV}$ luminosity (also corrected for absorption). The thin line and shaded region show the relation of these two quantities observed in Sy1 and QSOs (mean and $\pm 1 \sigma$ dispersion)

much higher than that estimated by Iwasawa (1999) and Turner (1999) in their faint hard X-ray spectrum. In the latter case the intrinsic X-ray luminosity could be much higher.

We should however mention that the sample presented in Paper I (Table 1) is probably biased in favor of objects which are particularly bright in the broad lines emission, just as a consequence of the selection criterion (i.e. those absorbed objects which show at least some broad emission lines). Nonetheless, it is highly unlikely that such a selection effect accounts for a factor of $\sim 100$ in the $\mathrm{H} \alpha$ flux.

It is worth noting that a diagram analogous to Fig. 1 was presented by Veilleux et al. (1999, therein Fig. 3) when discussing the detection of broad $\mathrm{Pa} \alpha$ in Ultraluminous Infrared Galaxies (ULIRGs); there the broad $\mathrm{H} \alpha$ is replaced by the broad $\mathrm{Pa} \alpha$ and the (intrisic) X-ray luminosity is replaced by the far-IR luminosity. From that diagram it can be similarly inferred that the broad component of the Pa $\alpha$ detected in ULIRGs cannot be ascribed to reflection. For what concerns Seyfert galaxies, Veilleux et al. (1997) reached a similar conclusion stating that the broad component of the hydrogen near-IR lines detected in some Sy2s cannot be ascribed to reflection.

Finally, also in the reflection scenario the lack of silicate absorption in Sy2s and of the lack of the carbon dip in reddened Sy1s is not explained.

\subsection{Reduction of the extinction scattering component}

Unlike the case of Galactic stars where both absorption and scattering contribute to the extinction, in obscured AGNs we may have effects of scattering back into our beam. This would imply a reduction of the scattering component in the effective extinction curve. However, the net effect must be small compared to what is observed for the objects of our sample. Even in the most extreme case that the absorbing/scattering medium covers the whole solid angle seeing by the nuclear source (that is very unlikely) and that the scattered radiation enters our beam without undergoing any dust absorption (that is also very unlikely), then the effect would be to completely remove the scattering component out of the total extinction curve. Since at the wavelengths of interest $\tau_{\text {scat }} \approx 2 \tau_{\text {abs }} \approx 2 / 3 \tau_{\text {ext }}$, the effect of this extreme case would be to reduce the extinction and reddening by a factor of 3 at most. While in our sample we observe several cases for which the reddening deficit is much higher.

The analysis above makes the implicit assumption that the line of sight is "typical", but it is possible that the absorbing column along the line of sight is lower than the scattering column along the other directions. In the latter case, and under the extreme assumption discussed above, the effective extinction could be reduced further. Yet, this effect of scattering back into the beam would not explain the lack of absorption features in the UV and in the mid-IR.

\subsection{Extended BLR}

Several reverberation mapping studies have shown that the BLR has a size of $\sim 0.01$ pc. More specifically $R \approx$ $0.02 L_{45}^{1 / 2}$ pc (Clavel et al. 1991; Peterson 1993; Salamanca et al. 1994). On the other hand, various pieces of evidence indicate that the obscuring torus has a size of $\sim 1 \mathrm{pc}$ or larger (Gallimore et al. 1997; Greenhill \& Gwinn 1997; Ford et al. 1997; Granato et al. 1997; Maiolino 2000, and references therein). Since the scale of the latter is so much larger than both the X-ray source and the BLR, the absorbing medium along the line of sight should be the same for both. However, for many of the objects in the sample presented in Paper I (Table 1) the broad lines have a width of about $2000 \mathrm{~km} \mathrm{~s}^{-1}$, which is somewhat narrower than usually observed in type 1 Seyferts and QSOs. This might imply that in these objects the BLR, or the fraction of it that we observe, is more extended than assumed and with dimensions comparable to the obscuring torus. As a consequence, the BLR might be observed, on average, through a lower absorbing column with respect to the nuclear X-ray source.

However, since the broad lines in many of these objects are weak, very broad wings might have been undetected because either lost in the noise or confused with the underlying continuum features, thus yielding to an underestimate of the real width of these lines. Also, in some of these objects the broad lines were observed to vary on time scales shorter than a few months, suggesting a BLR scale smaller than 0.1 pc (Eracleous \& Halpern 1993; Winkler et al. 1992; Rosenblatt et al. 1994; Giannuzzo \& Stirpe 1996; Peterson et al. 1982, 1984; Miller et al. 1979, 1985).

Summarizing, we cannot exclude that for some of the objects in our sample the BLR is extended enough that 
the broad lines and the (much smaller) hard X-ray source are obscured by columns of gas that are significantly different. However, this is unlikely to be the case for most of the objects.

Finally, the lack of the silicate feature in the mid-IR spectra of Sy2s and the lack of the carbon dip in the UV spectra of reddened Syls remain unexplained by the differential absorption scenario discussed in this section.

\subsection{Absorption by BLR clouds}

If one or more BLR clouds are located along the line of sight toward the X-ray nuclear source then they would contribute to the observed gaseous absorbing column density (BLR clouds are expected to have $N_{\mathrm{H}} \approx 10^{23} \mathrm{~cm}^{-2}$ ), but obviously this absorbing column would not affect the observed broad line flux. However, should the low $E_{B-V} / N_{\mathrm{H}}$ be a feature common to most AGNs and should the cause of this effect be absorption by BLR clouds, then this would require a large covering factor of the BLR clouds, at least as high as the covering factor of the obscuring medium $^{1}$, i.e. $\sim 80 \%$. On the other hand the covering factor of the BLR clouds is estimated to be low $(\sim 10 \%)$ based on the absence of any Ly-edge cutoff in the UV spectrum of QSOs. Therefore, absorption due BLR clouds might explain some of the cases of low $E_{B-V} / N_{\mathrm{H}}$, but probably not the majority of them.

Another reason to discard this explanation is related to the lack of strong and short term variability of the absorbing $N_{\mathrm{H}}$ in the X-rays. If most of the photoelectric cutoff observed in the hard X-rays is due to a single or a few BLR clouds along the line of sight, then a strong variation of the $N_{\mathrm{H}}$ would be expected on a time scale of a few weeks or less, given the expected size of the BLR clouds $(<0.01 \mathrm{pc})$ and their velocity. However, about $1 / 3$ of the objects in our sample were observed two or more times in the hard X-rays (e.g. Gilli et al. 2000; Malizia et al. 1997) and the $N_{\mathrm{H}}$ inferred from the cutoff does not show the strong variations expected from the motion of a BLR cloud passing on the line of sight. There are a few objects (most of them not in our sample) whose hard Xray monitoring has indeed shown variations of $N_{\mathrm{H}}$, but such variations are on time scales of years, i.e. consistent with a distance of the absorber from the nucleus of a few pc (Maiolino 2000). The only case known to show rapid $N_{\mathrm{H}}$ variability is NGC 4151.

Again, the issues related to the silicate feature and to the carbon dip remain unexplained also in this case.

\subsection{Dust sublimation}

The inner part of the obscuring torus is directly exposed to the UV radiation emitted by the nuclear source. If the UV flux at this location is large enough, the dust

\footnotetext{
${ }^{1}$ A covering factor of $80 \%$ for the obscuring torus is inferred both from the Sy2/Sy1 ratio and from the opening angle of the light cones observed in Sy2s (Maiolino \& Rieke 1995).
}

equilibrium temperature can reach the sublimation value (Laor \& Draine 1993; Phinney 1989). If dust is sublimated for a significant fraction of the absorbing gaseous column this would explain the reduced $E_{B-V}$ with respect to that expected from the observed $N_{\mathrm{H}}$. However, this model has problems when compared with the low covering factor inferred for the ionized gas in the circumnuclear region of AGNs, as discussed in the following.

The capability of the dust in competing with the gas in the absorption of UV ionizing photons increases significantly with the ionization parameter $U$. Indeed, a highly ionized gas is much more transparent than dust to the UV ionizing photons. In particular, for a Galactic gas-to-dust ratio and dust composition, dust grains are expected to dominate the absorption of photons beyond the Lyman edge when $U$ is higher than about $10^{-2}$. At the inner face of the torus the ionization parameter is expected to be about 0.1 or larger (Netzer \& Laor 1993; Netzer 2000) and therefore most of the UV ionizing photons should be absorbed by the dust which reprocesses this radiation into near and mid-IR emission, which is actually observed in the nuclear region of AGNs (e.g. Maiolino et al. 1995, 1998; Clavel et al. 2000; Thatte et al. 1997; Oliva et al. 1999). As a consequence, although the obscuring torus probably subtends as much as $80 \%$ of the solid angle seen by the nuclear UV source, very little line emission is produced. The emission lines that would be produced by the torus in absence of dust should have a width significantly smaller than the broad lines and close to the width of the narrow lines. The effect of strong absorption of UV ionizing photons from dust at the inner face of the torus has been proposed to explain the marked separation between BLR and NLR and to explain the low covering factor of the NLR. A more detailed discussion of this issue is given in Netzer \& Laor (1993), in Laor \& Draine (1993) and Pier \& Voit (1995). Here we do not discuss further the implications of dust absorption for the photoionization models of the circumnuclear gas, but we use these considerations as a constraint for the properties of the dust at the inner face of the torus.

If most of the gas in the torus is dust-free, especially at the inner face, because of dust sublimation, then photons at wavelengths shortward of the Ly edge should ionize the gas in the torus and should produce powerful hydrogen lines nearly as narrow as the narrow lines. The covering factor of the torus is estimated to be $\sim 80 \%$. Until a few years ago the covering factor of the NLR was estimated to be $\sim 2 \%$ (e.g. Netzer \& Laor 1993); however recent observations of the UV spectrum of QSOs indicate a shortage of ionizing photons with respect to former estimates by a factor of about 4 (Zheng et al. 1997; Laor et al. 1997) therefore implying a larger covering factor of the NLR $(\sim 8 \%)$, but which is still much lower than the covering factor estimated for the obscuring torus. As a consequence, the flux of the emission lines produced by the dust-sublimated torus should be about 10 times larger than the observed narrow lines. 
A possible way to suppress the line emission in the dust sublimated medium is to make the ionized gas very hot. However, in the latter case the absorbing medium observed in the hard X-rays should appear "warm" (according to the X-ray standards). On the other hand, in most of the objects of the sample presented in Paper I the absorbing gas is "cold" beyond doubt.

Summarizing, it is difficult to ascribe the low $E_{B-V} / N_{\mathrm{H}}$ ratio to dust sublimation at the inner face of the torus without running into a serious emission line budget problem.

For what concerns the absorption features, dust sublimation might explain the lack of the carbon dip at $2175 \AA$ in reddened Sy1s, since the very small grains which are responsible for this feature undergo sublimation more easily than larger grains. However, it is difficult to ascribe the lack of the silicate feature in the spectra of Sy2s to sublimation, indeed the absence of this absorption feature requires a dust distribution dominated by grains larger than a few $\mu \mathrm{m}$ (i.e. significantly larger than the maximum dimension of $0.25 \mu \mathrm{m}$ inferred for the Galactic medium), rather than the destruction of small grains (Sect. 3.3).

\subsection{Low dust-to-gas ratio}

One of the simplest ways to explain the observed low $E_{B-V} / N_{\mathrm{H}}$ is to assume that the dust-to-gas mass ratio in the obscuring medium is lower than the standard Galactic value. However, a reduced abundance of dust in the dense medium of the circumnuclear clouds is not obvious to justify. The vicinity of the AGN could help to create the conditions that act to destroy dust grains. In particular, sputtering by X-rays and/or shocks might be a possible mechanisms of dust destruction in this environment.

Obviously, the $E_{B-V} / N_{\mathrm{H}}$ ratio decreases linearly with the dust-to-gas ratio, if the dust properties remain unchanged. This is shown in the top panel of Fig. 2. The trend of $E_{B-V} / N_{\mathrm{H}}$ as a function of the dust-to-gas ratio relative to the Galactic value is given by the thick solid line. The horizontal thin line and shaded region show the mean and $\pm 1 \sigma$ dispersion of $E_{B-V} / N_{\mathrm{H}}$ for the objects in the sample presented in Paper $\mathrm{I}^{2}$. A factor of about 10 lower dust-to-gas ratio is required to match the mean of the observed $E_{B-V} / N_{\mathrm{H}}$ values. The thin dashed vertical lines indicate the range of gas-to-dust ratios that matches the observed $E_{B-V} / N_{\mathrm{H}}$ for $\sim 68 \%$ of the objects $( \pm 1 \sigma)$, which implies a dust-to-gas ratio between $\sim 3$ times and 50 times lower than Galactic. A dust underabundance of factor of $\sim 100$ is required to explain the lowest $E_{B-V} / N_{\mathrm{H}}$ found in Paper I (see Fig. 1 in that paper). The central panel shows the trend for $A_{V} / N_{\mathrm{H}}$, which also decreases linearly since the absorption curve was assumed constant.

As the dust content decreases, the absorption of UV ionizing photons by dust grains becomes gradually less

\footnotetext{
${ }^{2}$ In this analysis we excluded the three Low Luminosity AGNs and the three objects whose reddening was estimated by means of the continuum fit (Paper I).
}

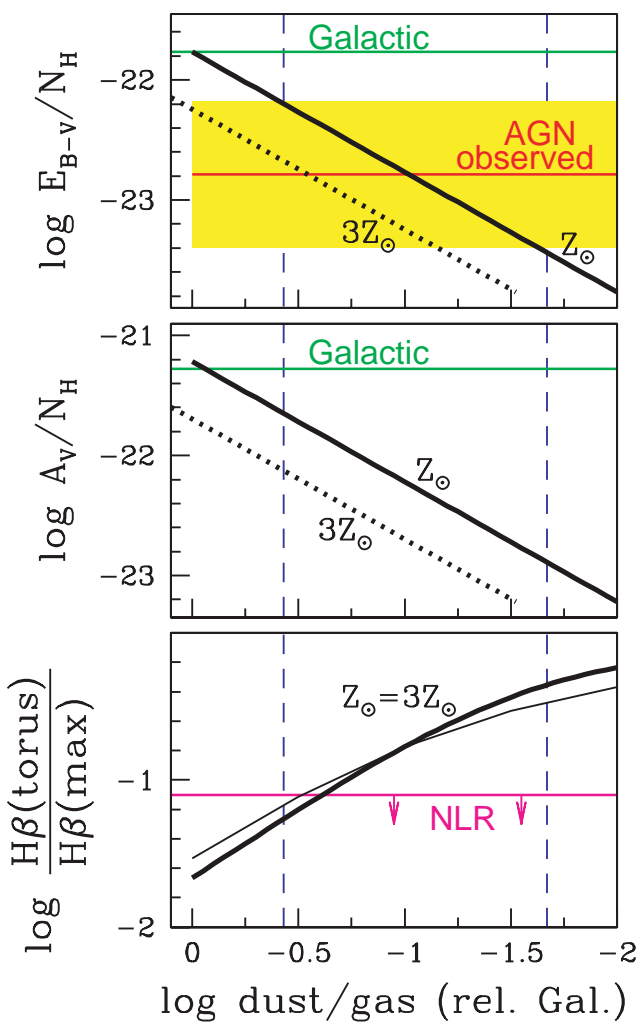

Fig. 2. Expected variation of extinction and emission line properties as a function of the dust-to-gas ratio relative to the Galactic value. The predictions of the models, which assume a standard dust size distribution, are shown by the thick solid lines. The upper two panels show the expected variation of the $E_{B-V} / N_{\mathrm{H}}$ and $A_{V} / N_{\mathrm{H}}$ ratios. The line and shaded area marked by "AGN observed" are the mean and $\pm 1 \sigma$ dispersion value found in the sample of Paper I. The thin dashed vertical lines indicate the range of gas-to-dust ratios that matches the observed $E_{B-V} / N_{\mathrm{H}}$ for $\sim 68 \%$ of the objects $( \pm 1 \sigma)$. The thick dotted lines indicate the effect of an increased dust-to-gas ratio due to a metallicity three times solar. In the bottom panel " $\mathrm{H} \beta$ (torus)" is the line luminosity produced within the dusty torus while " $\mathrm{H} \beta(\max )$ " is what one expects if all the ionizing photons are absorbed by the gas (i.e. the dust-free case). The light horizontal line constrains the maximum line emission produced by the torus (in terms of $\mathrm{H} \beta$ (torus) $/ \mathrm{H} \beta(\max )$ ) based on the maximum covering factor of the Narrow Line Region ( $\sim 8 \%)$. The thin solid line in the bottom panel shows, for comparison, the result obtained with Cloudy

important and, as discussed in the previous section, the emission of nebular lines gradually increases. In order to check whether the required dust depletion runs into the same emission line budget problem as in the case of the sublimated dust (Sect. 2.5), we have created a simple semi-analytical code to calculate the ionization structure and hydrogen lines emissivity of a gaseous cloud containing a variable amount of dust. We assumed a ionization parameter $U=0.1$ at the inner face of the cloud, as this is about the value expected at the inner face of the torus (Netzer \& Laor 1993). The covering factor was assumed to be $80 \%$.

The results of the model were then compared with the expected emission line flux in the case of a dust-free 
medium with a covering factor equal to unity, i.e. the maximum hydrogen line flux $(\mathrm{H} \beta(\max ))$ which can be obtained with the available photoionizing luminosity. The ratio between the hydrogen line flux in the dusty and in the dust-free cases gives the importance of the line emission from the obscuring torus relative to other gaseous clouds which are either free from dust, such as the BLR clouds, or whose ionization parameter is low enough to make unimportant the effect of dust absorption of UV ionizing photons, such as the NLR clouds. The thick black solid line in the bottom panel of Fig. 2 shows the ratio between the $\mathrm{H} \beta$ flux in the case of a dusty medium $(80 \%$ covering factor) and the case of a dust-free medium $(100 \%$ covering factor), $\mathrm{H} \beta$ (torus) $/ \mathrm{H} \beta$ (max), as a function of the dust-to-gas ratio in the obscuring torus.

We also performed the same calculation with Cloudy, the photoionization code provided by Ferland and collaborators (Ferland 1999), which accounts for several effects which are not included in our simple code. The output of Cloudy is overplotted with a thin solid line in the bottom panel of Fig. 2. Cloudy's result is not much different from ours and, partly, it is also to ascribe to the extinction curve used by Cloudy which is somewhat different from the Galactic standard curve (e.g. $R_{V}=A_{V} / E_{B-V}=4$, to be compared with the standard Galactic value of $R_{V}=3.1$ ). This comparison justifies us in using our code also for other models in the next section (Sect. 3). The advantage of our code is that it is possible to use an arbitrary dust composition with a (quasi-) continuous distribution of grain sizes.

The $\mathrm{H} \beta$ (torus) $/ \mathrm{H} \beta(\max )$ values obtained with the model discussed above have to be compared with the flux of the narrow lines. More specifically, the flux of the lines emitted by the torus should be lower than the flux of the narrow lines not to incur in the emission line budget problems discussed in Sect. 2.5; this implies that $\mathrm{H} \beta$ (torus) $/ \mathrm{H} \beta(\max )$ must be lower than the covering factor of the NLR clouds, i.e. $<0.08$ (Sect. 2.5). This limit is shown with an horizontal line in the bottom panel of Fig. 2. The dust-to-gas ratio required to match the mean observed $E_{B-V} / N_{\mathrm{H}}$ would give $\mathrm{H} \beta$ (torus) $/ \mathrm{H} \beta(\max ) \approx$ 0.15 that is inconsistent with the NLR covering factor. Although, higher values of $E_{B-V} / N_{\mathrm{H}}$ might be marginally consistent with the NLR emission, the values of $E_{B-V} / N_{\mathrm{H}}$ lower than the mean would imply an even higher $\mathrm{H} \beta$ (torus) $/ \mathrm{H} \beta(\max )$, as shown in Fig. 2.

In Sect. 2.1 we mentioned that the nuclear region of AGNs is generally characterized by super-solar metallicities. As we are going to discuss, a high metallicity helps to relax the constraints on the emission line flux. Metallicity variations change the dust-to-gas ratio, but the variation of the dust-to-gas ratio due to metallicity leaves unaffected the measured $E_{B-V} / N_{\mathrm{H}}$ and $A_{V} / N_{\mathrm{H}}$. As discussed in Sect. 2.1, this is because the $N_{\mathrm{H}}$ is measured in the hard X-rays through the column of metals. On the other hand, the increased dust-to-gas ratio, regardless of whether due to metallicity or to other effects, does affect the dust-to-gas opacity for the UV ionizing photons and, therefore, changes the $\mathrm{H} \beta$ (torus) $/ \mathrm{H} \beta$ (max) accordingly. As a consequence, even if metallicity does not affect the $E_{B-V} / N_{\mathrm{H}}$ ratio, it can "decouple" the latter ratio from the emission line budget constraints. In particular, the dotted lines in Fig. 2 show the effect of a metallicity three times higher than solar. On the top and central panel of Fig. 2 the (metallicity-) increased dust-to-gas ratio leaves unaffected the quantities on the $Y$-axis, thus yielding a shift on the $X$-axis. Instead, in the bottom panel the $\mathrm{H} \beta$ (torus) $/ \mathrm{H} \beta$ (max) ratio does change accordingly to the reduced dust-to-gas ratio, i.e. this ratio keep following the trend given by the solid line. As a result, for the same $E_{B-V} / N_{\mathrm{H}}$ ratio the implied $\mathrm{H} \beta$ (torus) $/ \mathrm{H} \beta$ (max) is significantly lower. In particular, at the dust-to-gas ratio which gives an $E_{B-V} / N_{\mathrm{H}}$ accounting for the mean observed value, $\mathrm{H} \beta$ (torus) $/ \mathrm{H} \beta(\max ) \approx 0.07$, which is marginally consistent with the covering factor of the NLR. Yet, for lower values of $E_{B-V} / N_{\mathrm{H}}$ the line emission from the torus is still much larger than the NLR.

Summarizing, a low gas-to-dust (mass) density ratio might probably explain the reduced $E_{B-V} / N_{\mathrm{H}}$ for several of the objects without incurring in emission line budget problems, especially if the metallicity is super-solar as claimed by several authors. However, low values of $E_{B-V} / N_{\mathrm{H}}$ (lower than the mean) are still difficult to explain with this model.

Certainly, the reduced dust-to-gas ratio alone cannot account for the lack of the silicate feature in the midIR spectra of Sy2, nor for the lack of the carbon dip in reddened Sy1s.

\section{The case for large dust grains}

\subsection{Plausibility of the large grains scenario}

So far we have adopted the standard extinction curve observed in the diffuse Galactic interstellar medium. However, the physical conditions of the gas in the nuclear region of Seyferts and QSOs are so extreme that there is no reason to assume that dust has the same properties as in the diffuse interstellar medium of our Galaxy. The gas densities of the obscuring medium in AGNs probably exceeds $10^{5}-10^{6} \mathrm{~cm}^{-3}, 3$ i.e. a density similar or higher than observed in the densest dark clouds of our Galaxy. Along the line of sight of dense Galactic molecular clouds the extinction curve is generally found to be flatter than in the diffuse ISM (Vrba \& Rydgren 1984; Carrasco et al. 1973; Whittet et al. 1976; Cardelli et al. 1989). Various observational evidences indicate that most likely such flat extinction curves are due to a distribution of grain sizes on average larger than in the diffuse ISM (Kim et al. 1994;

\footnotetext{
${ }^{3}$ Such a high density is inferred by the requirement of confining columns of gas higher than $10^{24}-10^{25} \mathrm{~cm}^{-2}$ in a region smaller than a few pc, to avoid a gas mass higher than the dynamical mass (Risaliti et al. 1999). The presence of clouds with densities of $10^{5}-10^{6} \mathrm{~cm}^{-2}$ in the circumnuclear region of AGNs is also directly inferred from $\mathrm{HCN}$ and other millimetric observations of AGNs with arcsecond resolution (e.g. Tacconi et al. 1994).
} 
Weingartner \& Draine 2000). Larger grains may form either by accretion from the gas phase or by grain coagulation. The former case should increase the $A_{V} / N_{\mathrm{H}}$ ratio, while the latter case should generally yield a reduced $A_{V} / N_{\mathrm{H}}$. Although measurements of $A_{V} / N_{\mathrm{H}}$ are very uncertain, some works suggest that $A_{V} / N_{\mathrm{H}}$ is lower in dense clouds characterized by flatter extinction curves, therefore supporting the coagulation scenario (Jura 1980; Kim \& Martin 1996). Also, theoretical considerations indicate that coagulation is much more effective for the growth of dust grains than accretion from the gas phase (Draine 2000).

Since the grain coagulation rate increases with density ( $\propto n^{1 / 2}$, Draine 1985), the effect is expected to be even more dramatic in the circumnuclear clouds of AGNs. In particular, for a gas density in excess of $10^{6} \mathrm{~cm}^{-2}$ the estimated time scale for the depletion of small grains due to coagulation is shorter than about $10^{5} \mathrm{yr}$, i.e. shorter than the typical dynamical time scale in the circumnuclear region of AGNs. This not only implies that dust grains have time to coagulate before being replaced by gas coming from the outer regions, but actually some mechanism that prevents grains to grow without limits must be invoked (e.g. shocks).

Summarizing, a dust distribution biased in favor of large grains, probably as a consequence of coagulation, is naturally expected in the extreme physical conditions of the gas in the circumnuclear region of AGNs.

The presence of large grains in the obscuring medium of AGNs was also proposed by Laor \& Draine (1993) in relation to the IR properties of active nuclei. In the following we discuss the effects of large grains, specifically formed by coagulation, on the observational quantities discussed in Paper I.

\subsection{The effect of large grains on $E_{B-V} / N_{H}$}

To obtain a more quantitative description of the effects of large grains in the circumnuclear region of AGNs we modelled the size grain distribution with a power-law:

$\mathrm{d} n(a) \propto a^{-\mathrm{q}} \mathrm{d} a, \quad a_{\min } \leq a \leq a_{\max }$

where $a$ is the grain diameter (assumed spherical) and $\mathrm{d} n(a)$ the density of grains with size between $a$ and $a+\mathrm{d} a$. Absorption and scattering cross sections were calculated by using the refractive indices in Draine \& Lee (1984) and in Laor \& Draine (1993), and by numerically solving the Mie formulas. The extinction curve in the Galactic diffuse ISM was successfully described by Mathis et al. (1977) with a mixture of graphite $(47 \%)$ and silicate $(53 \%)$ grains and with $a_{\min }=0.005 \mu \mathrm{m}, a_{\max }=0.25 \mu \mathrm{m}$ and $q=3.5^{4}$. The effect of large grains was investigated by

\footnotetext{
${ }^{4}$ Note that this model does not include the very small grains (PAHs) which are thought to be responsible for many of the emission feature in the mid-IR spectrum and probably contribute significantly also to the absorption dip at $2175 \AA$ (Weingartner \& Draine 2000).
}

us in various ways: either by increasing $a_{\max }$, or $a_{\min }$, or by decreasing $q$, or by a combination of these possibilities. Coagulation was assumed by imposing the total dust mass to be constant (relative to the gas mass). The increased average grain size always gives a flatter extinction curve. As long as $a_{\max }$ is larger than $0.5 \mu \mathrm{m}$, coagulation also yields to $E_{B-V} / N_{\mathrm{H}}$ and $A_{V} / N_{\mathrm{H}}$ lower than the Galactic standard value. We cannot discuss here all of the possible cases which give flatter extinction curves. In the following we discuss three representative families of models, all of which assume the same $a_{\min }=0.005 \mu \mathrm{m}$, but different $a_{\max }$ and $q$.

\subsection{1. $q=3.5$, increasing $a_{\max }$}

Figure 3 is analogous to Fig. 2, but in this case the dustto-gas (mass) ratio is kept constant, while $a_{\text {max }}$ increases along the abscissa and the other two dust parameters are kept constant at $q=3.5$ and $a_{\min }=0.005$.

In addition to the panels shown in Fig. 2, we also include in Fig. 3 a panel giving the variation of $R_{V}=$ $A_{V} / E_{B-V}$ (the larger is $R_{V}$ the flatter is the extinction curve). In this case $R_{V}$ increases up to $\sim 7$ for very large $a_{\text {max }}$. Such large values of $R_{V}$ imply that the absorption in the optical is higher than that estimated from the reddening assuming a Galactic extinction curve.

The $\mathrm{H} \alpha$ luminosities for the objects shown in Fig. 1 were corrected assuming $R_{V}=3.1$. If $R_{V}$ is higher than Galactic, then the $\mathrm{H} \alpha$ luminosities have been underestimated by the following quantity:

$\Delta \log \left(L_{\mathrm{H} \alpha}\right)=\frac{E_{B-V}}{2.5}\left(k R_{V}-3.1\right) 0.79$

where $1<k<1.26$ depending on the details of the extinction curve. For an average $E_{B-V} \approx 0.5$ the correction is significant, i.e. $\approx 0.7 \mathrm{in} \log L_{\mathrm{H} \alpha}$. Some of the objects in Fig. 1 do appear to be that or even more underluminous in $\mathrm{H} \alpha$ with respect to the Sy1+QSO relation, thus supporting the large grains scenario. However, other objects are not underluminous in $\mathrm{H} \alpha$. For some of them the required $L(\mathrm{H} \alpha)$ correction is small and therefore still consistent with the Sy1+QSO distribution, but for others the corrected $\mathrm{H} \alpha$ luminosity becomes uncomfortably high $(\sim 2 \sigma$ above the Sy1+QSO distribution). However, as discussed in Sect. 2.2 our sample is probably biased in favor of $\mathrm{H} \alpha$ bright sources and, therefore, the excess of $\mathrm{H} \alpha$ luminosity discussed above does not necessary rule out the large grains hypothesis for these objects.

The second panel of Fig. 3 shows the expected trend for $E_{B-V} / N_{\mathrm{H}}$ (thick solid line). This ratio has a steep decline as a function of $a_{\max }$. It should be noted that the locus of the values observed in AGNs (shaded area) is no longer constant as in Fig. 2, this is because we also took into account that the reddening estimate $E_{B-V}$ based on the broad line ratios (Paper I) changes with the extinction curve. The mean $E_{B-V} / N_{\mathrm{H}}$ of the AGNs in our sample can be explained with $a_{\max } \sim 30 \mu \mathrm{m}$. A much larger value 


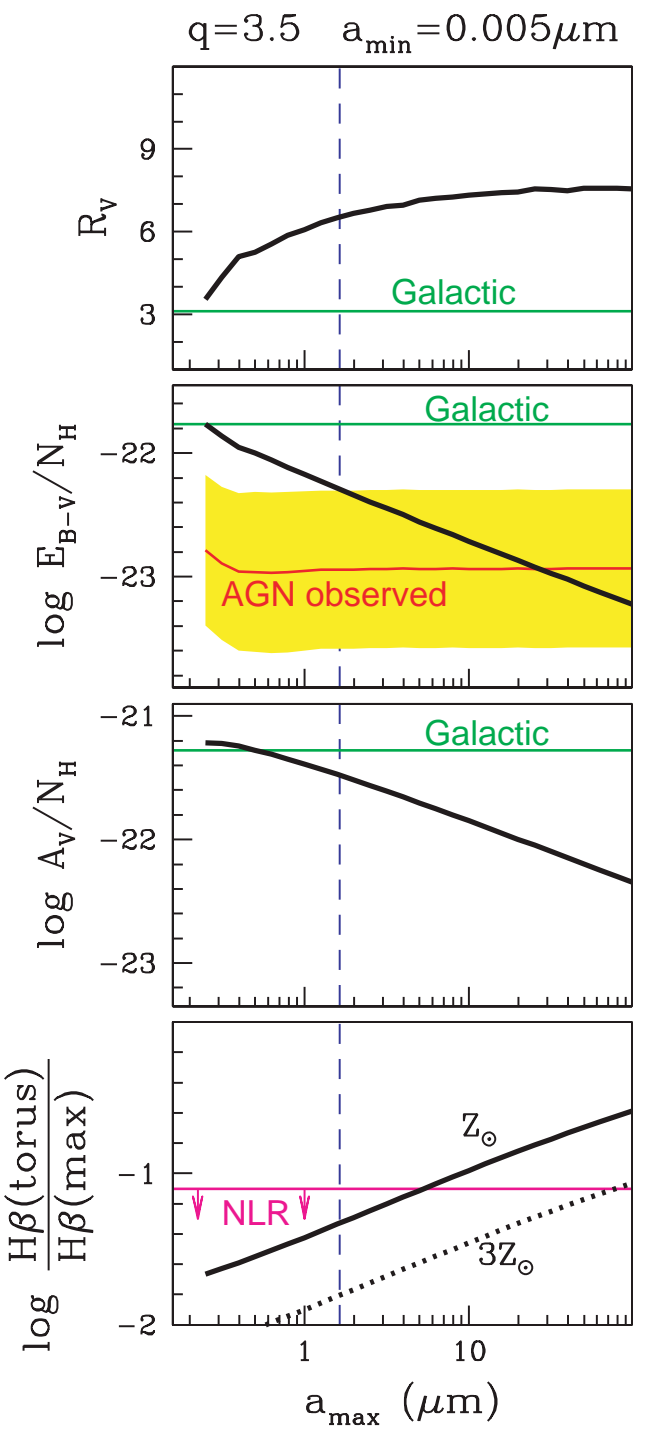

Fig. 3. Same as Fig. 2, but in this case the dust-to-gas ratio is constant while the maximum size of the grain distribution $a_{\text {max }}$ varies along the abscissa. The exponent of the grains size distribution $q$ and the minimum size $a_{\mathrm{min}}$ are kept constant to the standard values of 3.5 and $0.005 \mu \mathrm{m}$, respectively. In this figure we also show an additional panel, the top one, which show the variation of $R_{V}=A_{V} / E_{B-V}$. The thick dotted line in the bottom panel shows the effect of a higher dust-to-gas ratio due to a metallicity three times solar

of $a_{\max }(\geq 100 \mu \mathrm{m})$ is required to match the properties of objects with lower $E_{B-V} / N_{\mathrm{H}}$.

The ratio $A_{V} / N_{\mathrm{H}}$ also decreases rapidly with $a_{\max }$, indicating that large grains can, more generally, explain the low $A_{V} / N_{\mathrm{H}}$ inferred for many AGNs and discussed in Paper I.

The emission line budget problem does not improve much with respect to the case of low dust-to-gas ratio discussed in Sect. 2.6: for solar metallicities, the $\mathrm{H} \beta$ (torus) $/ \mathrm{H} \beta(\max )$ ratio ranges from $\sim 0.04$ up to $\geq 0.25$ for the values of $a_{\max }$ required to match the observed $E_{B-V} / N_{\mathrm{H}}$.
In the case of super-solar metallicities the dust-togas ratio increases, but both the extinction curve, and the $E_{B-V} / N_{\mathrm{H}}$ and $A_{V} / N_{\mathrm{H}}$ ratios remain unchanged, as discussed in Sect. 2.6. However, the $\mathrm{H} \beta$ (torus) $/ \mathrm{H} \beta$ (max) ratio decreases as a consequence of the increased dust absorption of UV ionizing photons (see discussion in Sect. 2.6). The case of an increased dust-to-gas ratio due to a metallicity three times higher than solar is shown with a dotted line in the bottom panel of Fig. 3. In this case the constraints given by the narrow line flux are met better and, in particular, for $a_{\max }$ lower than about $100 \mu \mathrm{m}$, the expected $\mathrm{H} \beta$ (torus) $/ \mathrm{H} \beta(\max )$ is lower than the NLR constraints. Yet, very low values of $E_{B-V} / N_{\mathrm{H}}$ (observed for some of the objects analyzed in Paper I) remain difficult to explain with this model.

\subsection{2. $a_{\max }=1 \mu \mathrm{m}$, decreasing $q$}

In Fig. 4 we show another "family" of grain distribution models: here $a_{\max }$ is kept constant and equal to $1 \mu \mathrm{m}$, while $q$ decreases along the abscissa. The extinction curve flattens very rapidly with $q$, as also indicated by the rapid growth of $R_{V}$. As shown, in the second panel, such flat extinction curve would explain the objects with nearly no reddening $E_{B-V}$, although substantial gaseous column $N_{\mathrm{H}}$ is observed along the line (Paper I). However, several of the objects considered in Paper I do show some reddening, therefore indicating that in these objects the extinction curve cannot be completely flat. A possibility, is that in the outer parts of the obscuring torus, where the gas density is probably much lower, the properties of dust are more similar to those of the diffuse Galactic ISM. In this case the extinction, curve should be nearly identical to Galactic, but shifted to higher values of absorption as a consequence of the higher density part of the torus which contributes with a flat extinction curve. The presence of "normal" dust in the outer parts obviously reduces $R_{V}$, but also increases $E_{B-V} / N_{\mathrm{H}}$. The case in which $15 \%$ of the gaseous absorbing column $N_{\mathrm{H}}$ contains "normal" dust is shown with a thick dashed line in Fig. 4. In this case $R_{V}$ is not unreasonably high, and the $A_{V}$ inferred from the observed reddening is still consistent with the presence of weak broad lines in the optical or IR spectra of the AGNs examined in Paper I. Yet, the large value of $R_{V}$ makes the $\mathrm{H} \alpha$ luminosity correction even higher than in Sect. 3.2.1, which complies with the $\mathrm{H} \alpha$-weak objects, but might be a problem for those objects which are above the distribution of Sy1+QSO in Fig. 1 (see analogous discussion in Sect. 3.2.1). Another problem with the inclusion of an even small (15\%) fraction of dust with Galactic extinction curve is that the ratio $E_{B-V} / N_{\mathrm{H}}$ has a much slower decline and, as shown in Fig. 4 , cases of very low $E_{B-V} / N_{\mathrm{H}}$ are difficult to explain.

Another important effect of this "family" of models is that, at variance with the $E_{B-V} / N_{\mathrm{H}}$ ratio, the $A_{V} / N_{\mathrm{H}}$ ratio is not reduced by a large factor, as shown in Fig. 4 . Therefore, this class of models does not provide the most 


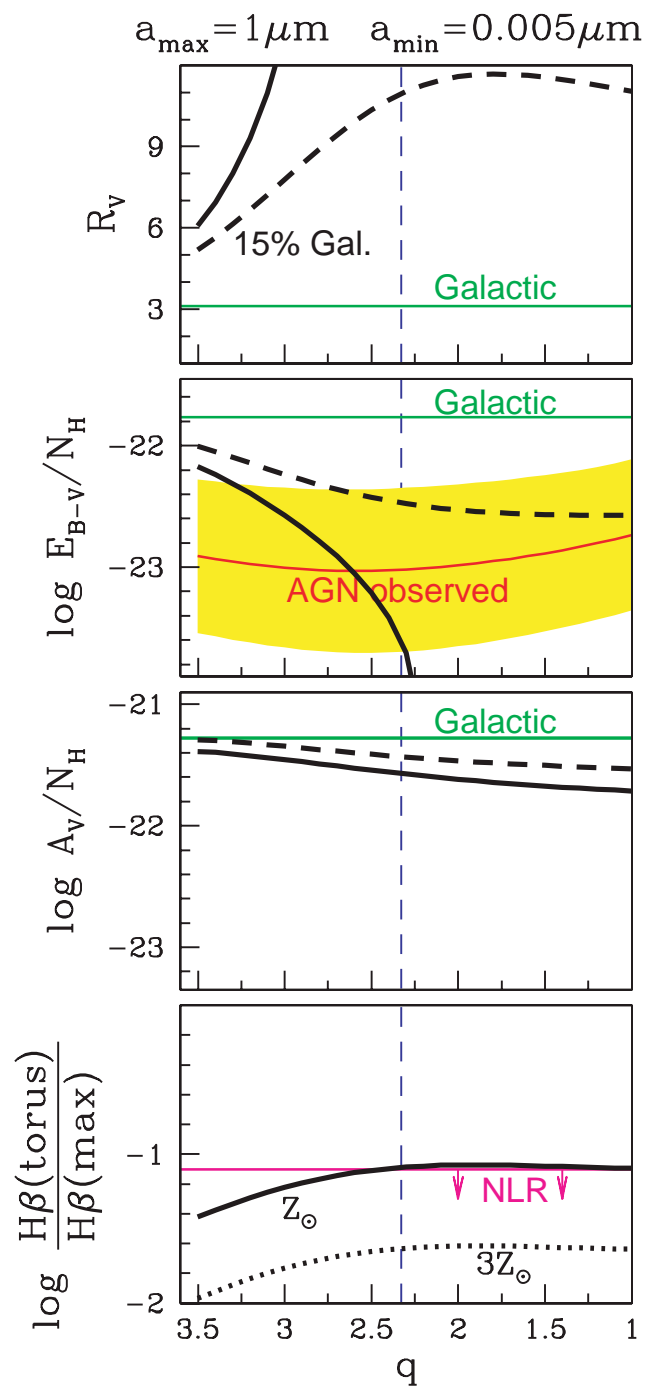

Fig. 4. Same as Fig. 3, but in this case the maximum size of the grain distribution $a_{\max }$ is kept constant and equal to $1 \mu \mathrm{m}$ while the exponent $q$ is varied along the abscissa. The thick dashed lines show the case in which $15 \%$ of the gaseous absorbing column (the outer part) contains Galactic standard dust

likely explanation for objects characterized by very low $A_{V} / N_{\mathrm{H}}$ values (Paper I).

Yet, one of the most appealing properties of this family of models is that the $\mathrm{H} \beta$ (torus) $/ \mathrm{H} \beta$ (max) ratio is low ( $\sim 8 \%$ at most) and may be much lower than the NLR constrain, especially if the dust-to-gas ratio is increased as a consequence of super-solar metallicity (bottom panel of Fig. 4). The value of $\mathrm{H} \beta$ (torus) $/ \mathrm{H} \beta$ (max) is obviously unaffected by a fraction of the column density containing "normal" Galactic dust, if the latter is located in the outer parts of the torus.

\subsection{3. $a_{\max }=10 \mu \mathrm{m}, q$ decreasing}

This family of models is shown in Fig. 5. These are similar to those discussed in Laor \& Draine (1993). Here we do not discuss in detail the properties of these models, but

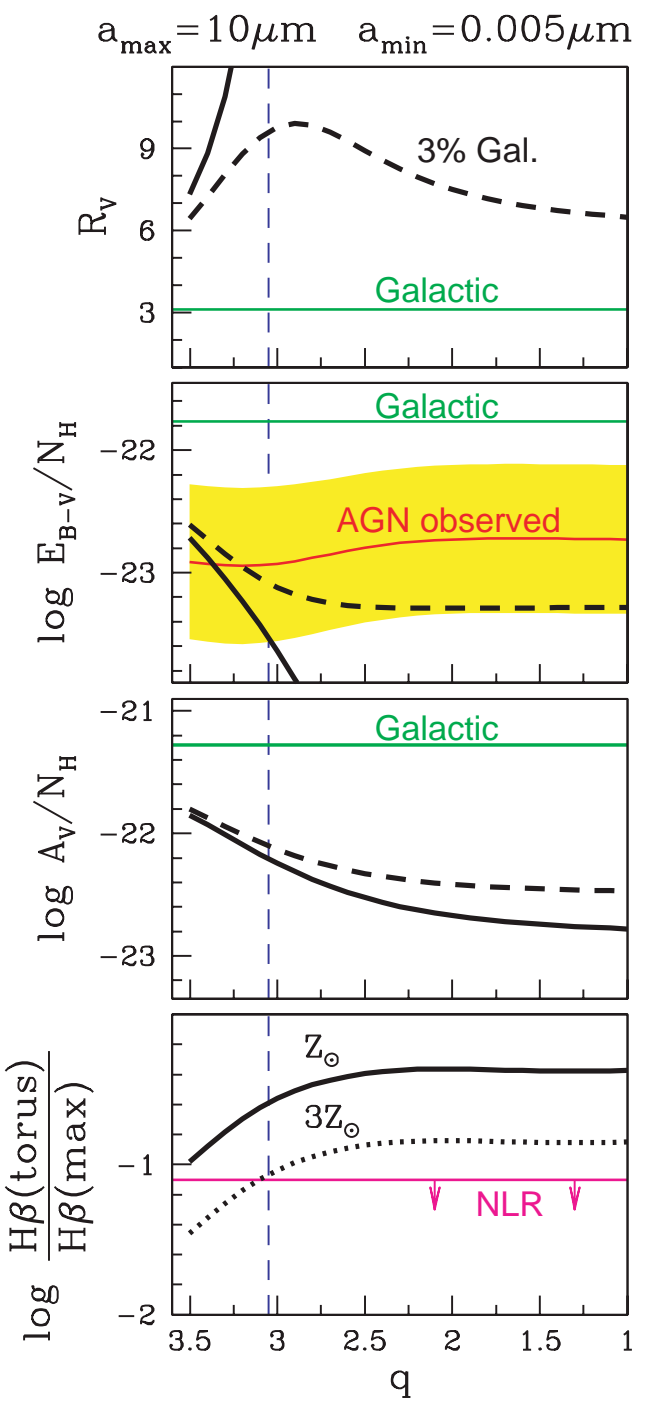

Fig. 5. Same as Fig. 4, but with $a_{\max }=10 \mu \mathrm{m}$. The thick dashed lines show the case in which $3 \%$ of the gaseous absorbing column (the outer part) contains Galactic standard dust

mostly focus on the main differences and similarities with the former case.

In these models the effect of decreasing $q$ not only rapidly flattens the extinction curve, but also decreases significantly the absorption $A_{V}$, and therefore can easily explain little reddened objects with low $A_{V} / N_{\mathrm{H}}$ (Paper I). If the metallicity is three times solar, or higher, the range of $q$ required to match the $E_{B-V} / N_{\mathrm{H}}$ distribution of most objects $(q>3.1)$ also complies with the NLR constraint on the torus emission line flux (bottom panel of Fig. 5).

As a consequence of the much reduced absorption $A_{V}$, the inclusion of an outer layer of Galactic dust reduces much more $R_{V}$ and affect much less $E_{B-V} / N_{\mathrm{H}}$. This is shown by the thick dashed line in Fig. 5 , which gives the case with only $3 \%$ of the (outer) column of gas containing Galactic "standard" dust. Therefore, this family of models relaxes the problems on the large $R_{V}$ discussed in the family of models discussed in Sect. 3.2.2: it gives very low $E_{B-V} / N_{\mathrm{H}}$ and also $A_{V} / N_{\mathrm{H}}$ values without incurring in large values of $R_{V}$. 


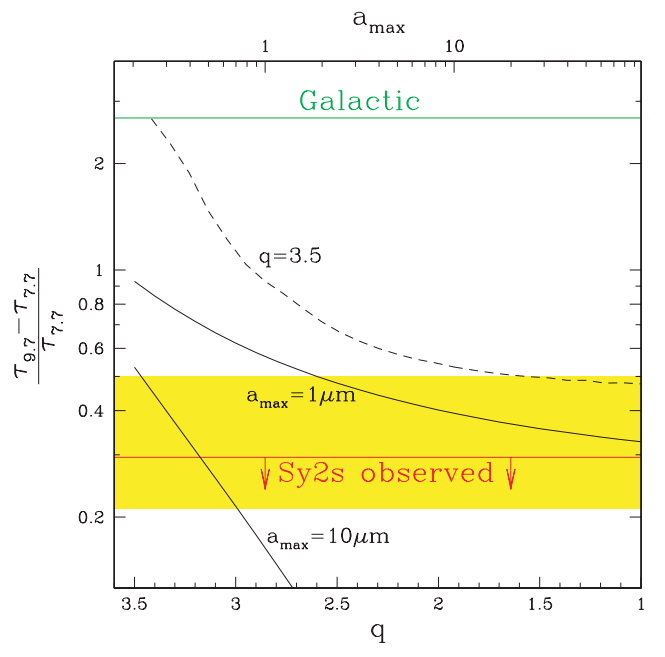

Fig. 6. Depth of the silicate feature relative to the featureless mid-IR absorption as measured by ratio $\left(\tau_{9.7}-\tau_{7.7}\right) / \tau_{7.7}$. The upper horizontal line gives the Galactic value, while the lower horizontal line gives the upper limit obtained for Sy2s (the shaded area gives the spread of the upper limits). The upper dashed curve gives the ratio expected by the model presented in Sect. 3.2.1 $(q=3.5)$ and is related to the upper abscissa scale, while the lower solid curves give the ratio expected by the models presented in Sects. 3.2.2 and 3.2.3 $\left(a_{\max }=1 \mu \mathrm{m}\right.$ and $\left.a_{\max }=10 \mu \mathrm{m}\right)$ and are related to the lower abscissa scale

\subsubsection{Summary of the effects on $E_{B-V} / N_{H}$}

We cannot describe here all of the dust models that matches the specific constraints for each single object. However, for most of the AGNs discussed in Paper I one or more dust distribution models match the specific constraints on $E_{B-V} / N_{\mathrm{H}}, E_{B-V}$, broad lines luminosity and detection.

\subsection{The effect of large grains on the silicate feature}

As mentioned in the introduction and discussed more in detail in Paper I, Sy2s appear characterized by significant mid-IR absorption which, nonetheless, is not associated to a deep silicate absorption feature at $9.7 \mu \mathrm{m}$ which is expected by a Galactic extinction curve. Since this silicate feature is produced by grains smaller than a few $\mu \mathrm{m}$, a dust distribution dominated by large grains can also explain this "anomaly" of the mid-IR spectra of Sy2s, as discussed in the following.

We take the optical depth at $7.7 \mu \mathrm{m}$ as representative of the featureless absorption in the vicinity of the silicate feature. We specifically choose this wavelength because it is the location of the PAH whose equivalent width is used by Clavel et al. (2000) to determine the mid-IR absorption. The difference of the optical depths $\tau_{9.7}-\tau_{7.7}$ is an alternative way to measure the equivalent width of the silicate feature, as long as it is not saturated. The ratio $\left(\tau_{9.7}-\tau_{7.7}\right) / \tau_{7.7}$ is a measure of the depth of the silicate feature relative to the featureless mid-IR absorption. In Fig. 6 the upper horizontal line indicates the standard Galactic value of this ratio, while the lower horizontal line indicates the (mean) upper limit of this ratio for Sy2s, as inferred from the data in Clavel et al. (2000), and the shaded area gives the spread of the upper limits (as inferred from the spread of the EW of the PAH at $7.7 \mu \mathrm{m}$ ). Figure 6 illustrates in different terms what was already discussed in Paper I: the upper limit on the depth of the silicate feature in the average spectrum of Sy2s is about one order of magnitude lower than expected from the Galactic extinction curve. The curves plotted in the diagram show the expected $\left(\tau_{9.7}-\tau_{7.7}\right) / \tau_{7.7}$ ratio according to the coagulation models discussed in Sect. 3.2. In particular, the dashed line refers to the model presented in Sect. 3.2.1 ( $q$ constant and equal to 3.5) and is related to the upper abscissa scale, while the solid curves refer to the models presented in Sects. 3.2.2 and 3.2.3 ( $a_{\max }$ constant) and are related to the lower abscissa scale. All of these models predict a depth of the silicate feature significantly lower than Galactic. The model with $a_{\max }=10 \mu \mathrm{m}$ can easily fit the constraint it given by the mid-IR observations, with $q$ just slightly lower than the canonical value of 3.5 .

As mentioned in Paper I (Sect. 4.1), the inner (hotter) mid-IR emitting region probably is not featureless but is also characterized by the $9.7 \mu \mathrm{m}$ feature in emission. Since the upper limit on $\tau_{9.7}-\tau_{7.7}$ was determined assuming an intrinsic featureless continuum, the possible presence of the $9.7 \mu \mathrm{m}$ feature in emission should relax such an upper limit. However, as mentioned in Paper I, the equivalent width of the $9.7 \mu \mathrm{m}$ emission feature is so small that the upper limit on $\left(\tau_{9.7}-\tau_{7.7}\right) / \tau_{7.7}$ would increase by a quantity of 0.15 at most, which certainly cannot account for the the discrepancy with the value expected for the Galactic case (Fig. 6).

Finally, we wish to emphasize that the presence of strong PAH features in the ISO spectra of Seyfert galaxies is not in contractdiction with our large grain model, since such PAH features most likely come from the host galaxy (where the dust properties are probably similar to the Galactic ISM) and not from the nuclear region.

\subsection{The effect of large grains on the carbon dip}

In Sects. 3.2 and 3.3 we only considered the effects of coagulation by increasing $a_{\max }$ and by decreasing $q$, since these are the parameters which primarily affect $R_{V}, E_{B-V} / N_{\mathrm{H}}$, $A_{V} / N_{\mathrm{H}}$ and the silicate feature. The latter quantities are instead little sensitive to $a_{\min }$. However, this is not the case for the carbon dip which, in the model of Mathis et al. (1977) is ascribed to graphite grains with sizes in the range $100 \AA-200 \AA$. In particular, the carbon dip is washed away from the extinction curve if coagulation depletes small grains by increasing $a_{\text {min }}$ above $200 \AA$. This is shown in Fig. 7, where we plot the variation in the UV shape extinction curve by modifying the dust distribution to have $a_{\min }=0.03 \mu \mathrm{m}$ and leaving $a_{\max }$ and $q$ to the standard values of 0.25 and 3.5 , respectively.

Note that recent studies have shown that most of the profile of the $2175 \AA$ dip is probably produced by even 


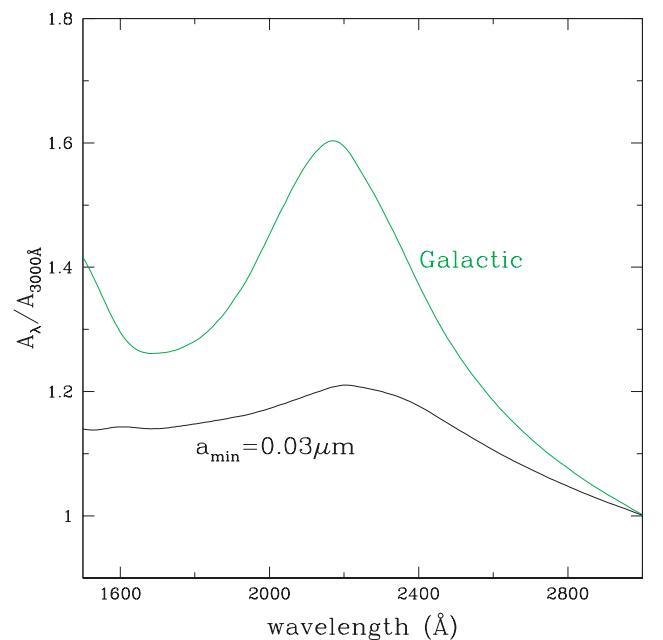

Fig. 7. Comparison of the Galactic UV extinction curve with the extinction curve given by a dusty medium which is depleted of grains smaller than $0.03 \mu \mathrm{m}$. Note the absence of the carbon dip in the latter case

smaller grains (PAHs, Weingartner \& Draine 2000) that are not included in our model. Obviously, in this case the depletion of small grains onto larger grains through coagulation would be even more effective in removing the $2175 \AA$ dip.

As a consequence, the coagulation scenario is also supported by the lack of a prominent carbon dip in spectra of reddened Sy1s (Paper I). However, the depletion of small grains can also be achieved with other physical processes (e.g. sublimation, Sect. 2.5) and, therefore, the absence of the carbon dip alone cannot be used to rule out other scenarios. Yet, whatever is the mechanism responsible for depleting small grains, the absence of the carbon dip certainly indicates that the properties of dust in AGNs are different from Galactic and, in particular, that the dust distribution is biased in favor of large grains.

\section{Conclusions}

We have discussed various interpretations of the observational evidences for "anomalous" properties of dust in the circumnuclear region of AGNs and, in particular, the reduced $E_{B-V} / N_{\mathrm{H}}$, the reduced $A_{V} / N_{\mathrm{H}}$, the absence of the silicate absorption feature in the mid-IR spectra of Sy2s and the absence of the carbon dip in the UV spectra of reddened Sy1s, which are reported in a companion paper (Maiolino et al. 2001, Paper I).

A dust distribution dominated by large grains as a consequence of coagulation is the interpretation which best fit the various observational constraints. The formation of large grains is naturally expected in the high density environment characterizing the circumnuclear region of AGNs. A dust grain distribution biased in favor of large grains makes the extinction curve flatter than Galactic and featureless (especially for what concerns the silicate mid-IR feature and the UV carbon dip). Coagulation also yields a reduced $E_{B-V} / N_{\mathrm{H}}$ and $A_{V} / N_{\mathrm{H}}$.
It should be noted that nearly all of the papers, published so far, dealing with the effects of dust in AGNs have adopted a standard Galactic extinction curve (essentially with the only exception of Laor \& Draine 1993, who first proposed the large grain scenario). The assumption that the properties of dust in the extreme conditions of the circumnuclear region of AGNs are similar to the Galactic diffuse interstellar medium is somewhat naive, given that even in the denser clouds of our Galaxy the extinction curve is already different with respect to the diffuse medium. Probably, many of the past studies considering the effects of dust obscuration and scattering in AGNs should be revised at the light of the findings obtained in this paper.

With regard to the scattered radiation (especially in obscured AGNs) it is worth noting that a dust distribution dominated by large grains would yield a nearly gray reflection, thus mimicking the scattering by free electrons. Large grains produced by dust coagulation would have a reduced scattering efficiency with respect to the standard Galactic dust mixture. However, while in some cases coagulation can result in a reduction of the scattering efficiency by more than an order of magnitude, for some of the dust grain distributions producing a flat extinction and scattering curve the reduction in the scattering efficincy is much lower (e.g. for the model discussed in 3.2.2 the scattering efficiency is reduced by a factor of $\sim 2$ ). Therefore, it is possible that some of the gray-reflected spectra found in some obscured AGNs, and ascribed to electron scattering, might actually be produced by large grains scattering.

We have also examined other possible interpretations for the "anomalous" dust properties discussed in Paper I, and more specifically:

1) a metallicity higher than solar (as often found in the central region of AGNs) might affect the estimate of the equivalent $N_{\mathrm{H}}$ from the photoelectric cutoff in the hard X-rays;

2) the observed broad lines might be scattered by a reflecting medium through a path of much lower gaseous column than the X-rays;

3) reduction of the scattering component of the extinction curve as a consequence scattering of the radiation into our beam;

4) the BLR might have a size comparable to that of the absorbing medium (at variance with what assumed by the standard unified model) and, therefore, suffer less absorption with respect to the nuclear X-ray source;

5) the hard X-rays might be partly absorbed by the same clouds which produce the broad lines;

6) the sublimation of dust at the inner face of the obscuring torus would produce a gaseous dust-free region;

7) the absorbing medium might simply be characterized by a low dust-to-gas ratio (in terms of mass).

Although some of these cases might apply to some of the objects examined in Paper I, none of them can fit all of the observational constraints for most of the objects, at variance with the large grains scenario. 
Acknowledgements. We are grateful to B. Draine for enlightening discussions during the early stages of this work. This paper also benefits of useful comments from A. Natta, M. Walmsley and from the referee R. Antonucci. This work was partially supported by the Italian Space Agency (ASI) under grant ARS99-15 and by the Italian Ministry for University and Research (MURST) under grant Cofin98-02-32. We thank G. Ferland for making Cloudy available to the community.

\section{References}

Barth, A. J., Filippenko, A. V., \& Moran, E. C. 1999, ApJ, 525,673

Berriman, G. 1989, ApJ, 345, 713

Brindle, C., Hough, J. H., Bailey, J. A., et al. 1990, MNRAS, 244,577

Cardelli, J. A., Clayton, G. C., \& Mathis, J. S. 1989, ApJ, 345, 245

Carrasco, L., Strom, S. E., \& Strom, K. M. 1973, ApJ, 182, 95

Clavel, J., Reichert, G. A., Alloin, D., et al. 1991, ApJ, 366, 64

Clavel, J., Schulz, B., Altieri, B., et al. 2000, A\&A, 357, 839

Corbett, E. A., Robinson, A., Axon, D. J., Young, S., \& Hough, J. H. 1998, MNRAS, 296, 721

Dietrich, M., \& Wilhelm-Erkens, U. 2000, A\&A, 354, 17

Draine, B. T. 1985, in Protostars and Planets II, ed. D. C. Black, \& M. S. Matthews, Sp. Sc. Ser. (The Univ. of Arizona Pr.)

Draine, B. T. 2000, in Encyclopedia of Astronomy \& Astrophysics, IOP and MacMillan [astro-ph/0008150]

Draine, B. T., \& Lee, H. M. 1984, ApJ, 285, 89

Eracleous, M., \& Halpern, J. P. 1993, ApJ, 409, 584

Ferland, G. J. 1999, in Astrophysical Plasmas: Codes, Models, and Observations, ed. J. Arthur, N. Brickhouse, \& J. Franco, Rev. Mex. Astron. Astrof. (Ser. Conf.), 9, 153

Ferland, G. J., Baldwin, J. A., Korista, K. T., et al. 1996, ApJ, 461,683

Ford, H., Tsvetanov, Z., Ferrarese, L., et al. 1997, in IAU Coll. 163, ASP Conf. Ser. 121, 620

Fosbury, R. A. E., Vernet, J., Villar-Martin, M., et al. 1999, in ESO Conference on Chemical Evolution from Zero to High Redshift, ed. J. Walsh, \& M. Rosa, ESO Astrophys. Symp. [astro-ph/9901115]

Gallimore, J. F., Baum, S. A., \& O'Dea, C. P. 1997, Nat, 388, 852

Giannuzzo, E. M., \& Stirpe, G. M. 1996, A\&A, 314, 419

Gilli, R., Maiolino, R., Marconi, A., et al. 2000, A\&A, 355, 485

Goodrich, R. W. 1992, ApJ, 399, 50

Goodrich, R. W., \& Miller, J. S. 1994, ApJ, 434, 82

Granato, G. L., Danese, L., \& Franceschini, A. 1997, ApJ, 486, 147

Greenhill, L. J., \& Gwinn, C. R. 1997, Ap\&SS, 248, 261

Hamann, E., \& Ferland, G. 1993, ApJ, 418, 1

Hamann, E., \& Ferland, G. 1999, ARA\&A, 37, 487

Iwasawa, K. 1999, MNRAS, 302, 96

Jura, M. 1980, ApJ, 241, 965

Kay, L. E. 1994, ApJ, 430, 196

Kim, S.-H., \& Martin, P. G. 1996, ApJ, 462, 296

Kim, S.-H., Martin, P. G., \& Hendry, P. D. 1994, ApJ, 422, 164

Laor, A., \& Draine, B. T. 1993, ApJ, 402, 441

Laor, A., Fiore, F., Elvis, M., Wilkes, B. J., \& McDowell, J. C. 1997, ApJ, 477, 93
Maccacaro, T., Perola, G. C., \& Elvis, M. 1982, ApJ, 257, 47

Maiolino, R. 2000, in X-ray astronomy 1999, ed. G. Malaguti, G. G. C. Palumbo, \& N. White, Astroph. Lett. Comm., in press [astro-ph/0007473]

Maiolino, R., \& Rieke, G. H. 1995, ApJ, 454, 95

Maiolino, R., Ruiz, M., Rieke, G. H., \& Keller, L. D. 1995, ApJ, 446, 561

Maiolino, R., Krabbe, A., Thatte, N., \& Genzel, R. 1998, ApJ, 493, 650

Maiolino, R., Marconi, A., Salvati, M., et al. 2001, A\&A, 365, 28 (Paper I)

Malizia, A., Bassani, L., Stephen, J. B., Malaguti, G., \& Palumbo, G. G. C. 1997, ApJS, 113, 311

Mathis, J. S., Rumpl, W., \& Nordsieck, K. H. 1977, ApJ, 217, 425

Miller, H. R. 1979, A\&AS, 35, 387

Miller, H. R. 1985, A\&AS, 59, 367

Morrison, R., \& McCammon, D. 1983, ApJ, 270, 119

Netzer, H. 2000, in X-ray astronomy 1999, ed. G. Malaguti, G. G. C. Palumbo, \& N. White, Astroph. Lett. Comm., in press

Netzer, H., \& Laor, A. 1993, ApJ, 404, L51

Oliva, E., Origlia, L., Maiolino, R., \& Moorwood, A. F. M. 1999, A\&A, 350, 9

Peterson, B. M. 1993, PASP, 105, 247

Peterson, B. M., Foltz, C. B., Byard, P. L., \& Wagner, R. M. 1982, ApJS, 49, 469

Peterson, B. M., Crenshaw, D. M., Meyers, K. A., Byard, P. L., \& Foltz, C. B. 1984, ApJ, 279, 529

Phinney, E. S. 1989, in Theory of Accretion Disks, ed. F. Meyer, W. J. Duschl, J. Frank, \& E. Meyer-Hofmeister, NATO ASI series (Kluwer), 457

Pier, E. A., \& Voit, G. M. 1995, ApJ, 450, 628

Reichert, G. A., Mushotzky, R. F., Holt, S. S., \& Petre, R. 1985, ApJ, 296, 69

Reynolds, C. S., Fabian, A. C., \& Inoue, H. 1995, MNRAS, 276,1311

Risaliti, G., Maiolino, R., \& Salvati, M. 1999, ApJ, 522, 157

Rosenblatt, E. I., Malkan, M. A., Sargent, W. L. W., \& Readhead, A. C. S. 1994, ApJ, 93, 73

Salamanca, I., Alloin, D., \& Baribaud, T. 1994, A\&A, 282, 742

Tacconi, L. J., Genzel, R., Blietz, M., et al. 1994, ApJ, 426, L77

Thatte, N., Quirrenbach, A., Genzel, R., Maiolino, R., \& Tecza, M. 1997, ApJ, 490, 238

Turner, T. J. 1999, ApJ, 511, 142

Veilleux, S., Goodrich, R. W., \& Hill, G. J. 1997, ApJ, 477, 631

Veilleux, S., Sanders, D. B., \& Kim, D.-C. 1999, ApJ, 522, 139

Vrba, F. J., \& Rydgren, A. E. 1984, ApJ, 283, 123

Young, S., Hough, J. H., Efstathiou, A., et al. 1996, MNRAS, 281, 1206

Whittet, D. C. B., van Breda, I. G., \& Glass, I. S. 1976, MNRAS, 177, 625

Wills, B. J., Wills, D., Breger, M., Antonucci, R. R. J., \& Barvainis, R. 1992, ApJ, 398, 454

Weingartner, J. C., \& Draine, B. T. 2000, ApJ, in press [astro-ph/0008146]

Winkler, H., Glass, I. S., van Wyk, F., et al. 1992, MNRAS, 257,659

Zheng, W., Kriss, G. A., Telfer, R. C., Grimes, J. P., \& Davidsen, A. F. 1997, ApJ, 475, 469 